Review

\title{
Intercellular Signaling in Cancer-the SMT and TOFT Hypotheses, Exosomes, Telocytes and Metastases: Is the Messenger in the Message?
}

\author{
John Smythies ${ }^{凶}$ \\ Center for Brain and Behavior, Department of Psychology, University of California, San Diego, USA \\ $\triangle$ Corresponding author: e-mail: jsmythies@ucsd.edu; web address: www.johnsmythies.com \\ () 2015 Ivyspring International Publisher. Reproduction is permitted for personal, noncommercial use, provided that the article is in whole, unmodified, and properly cited. \\ See http://ivyspring.com/terms for terms and conditions.
}

Received: 2015.04.10; Accepted: 2015.05.05; Published: 2015.05.23

\begin{abstract}
This review examines the current two leading hypotheses relating to cancer neogenesis-the somatic mutation theory (SMT) and the tissue organization field theory (TOFT) - and focuses on four specific issues.

What are the details of the process that changes the epigenetic cargo of the exosomes a cell produces when it becomes malignant?

Can exosomes produced by a malignant cell induce on their own a metastatic cancer in the target tissue?

What is the functional significance of the fact that exosomes from cancer cells carry in their loads segments of genomic DNA bearing cancer-related mutations across the entire spectrum?

What is the evolutionary advantage for the organism of the production by its cancer cells of exosomes that carry epigenetic instructions for the building of elaborate molecular mechanisms that promote the growth of metastatic cancers?

These issues are examined with a view of determining the support they give to one or other of the two hypotheses. The conclusion is that they support a specific form of TOFT in which exosomes play a key role.
\end{abstract}

Key words: Cancer, TOFT, SMT, exosomes, telocytes, oncosomes, metastases, bioelectric fields, non-linear dynamics, metastatic niche, evolution

\section{Introduction}

There are at present two quite different theories as to how cancers are caused - the classical somatic mutation theory (SMT) and the tissue organization field theory (TOFT). This paper will describe these theories, delineate four themes relating to them, and then determine how the two theories account for them and explain their significance for theories of cancer neogenesis. These themes are,

1. What are the details of the process that changes the epigenetic cargo of the exosomes a cell produces when it becomes malignant?
2. Can exosomes produced by a malignant cell induce on their own a metastatic cancer in the target tissue? This is on a line with the finding that a particular bioelectric event, by itself, can start a fully metastatic cancer.

3. What is the functional significance of the fact that exosomes from cancer cells carry in their loads segments of genomic DNA bearing cancer-related mutations across the entire spectrum?

4. What is the evolutionary advantage for the organism of the production by its cancer cells of exo- 
somes that carry epigenetic instructions for the building of elaborate molecular mechanisms that promote the growth of metastatic cancers?

\section{The somatic mutation theory}

The dominant theory of cancer genesis today is the Somatic Mutation Theory (SMT). This postulates only one causative abnormality i.e. mutated gene(s) in a cell's DNA. This change and its effects, plus a number of modulations by external factors, is supposed to account for the entire complex process of formation, growth and metastasis of the tumour. Each metastasis consists of a tumour cell that leaves the primary tumour and travels by the blood or lymph to attach to and invade other tissues. The role of the tumour-derived exosomes is essentially to provide a niche for optimum growth of the metastatic tumour cell.

\section{The tissue organization field theory (TOFT)}

Over the years an alternative theory of cancer genesis has been developed - the tissue organization field theory (TOFT) (1-6). This suggests that cancer results from a disorder of the microenvironment of the cell that represents the physico-chemical support by the morphogenetic field that drives epithelial cells (in particular) towards differentiation and phenotype transformation according to the systems biology rules that govern non-linear dynamic self-organizing structures. This involves complex and reciprocal biophysical, and biochemical communication between mesenchymal (somal or connective tissue) and parenchymal (epithelial) cells. In this process, gradients of bioelectric membrane resting potentials play a key role. The connection between electrical potentials and morphogenesis depends on the physical fact that flowing currents produce electromagnetic fields, and the lines of force of these fields determine the orientation and movement of all charged particles within the field including the movement of ions across membranes (2). Cancer is associated with an abnormal depolarization of these bioelectric fields, triggered by a carcinogen, and a resulting disorder in inter-and intra-cellular communication. Oncogene expression cannot form tumors if this depolarization is prevented (5). Recently Blackiston et al. (7) have shown that depolarization of a glycine receptor-expressing channel in Xenopus Laevis neural crest can induce a full metastatic melanoma with no involvement of any mutation, carcinogen, or DNA. Such interactions may also include protein interaction networks (PINs) that become rewired during the oncogenic process (8). The conclusions are that oncogenes do not cause cancer unless the environment becomes permissive, and that the environment can induce cancer without any oncogene or carcinogen. An interesting supportive observation is a tumour inserted into the regenerating limb of a salamander gets reprogrammed back into normal tissue. EM evidence for the presence of fractal patterns - that signal the presence of chaotic activity within the cell-on the surface of precancerous cells has been reported by Do1 et al. $(9,10)$.

A) lucid account of the TOFT theory has been presented by Tarin (11). He notes that intercellular signaling in tissues is a highly interactive, kinetic and adaptable non-linear process mediated by a complex of flexible networks composed of electrical, chemical and physical components. Most common cancers are not primarily caused by genetic or chromosomal lesions, but by a sustained failure to communicate between interacting cell lineages living in the complex society of the organism induced by many different primary agents - bioelectric, chemical, imbalances of hormones and other signaling molecules, ROS, implanted foreign bodies, bacteria and viruses, mutations, etc. (11).

These failures of communication initiate the formation of local disorderly, or rogue, cell groups. These groups wax and wane driven by the non-linear dynamics of the group as well as external factors. A sustained local disturbance of such interactions between different cell populations composing a tissue or organ can lead to disorderly arrangement and proliferation of a subpopulation of cells and to disturbances in the microstructure of the region. If one these corrupted groups, by chance, triggers a mechanism that attracts, recruits and incorporates into the group adjacent non-neoplastic cells, this may start the formation of a cancer (11).

Three of the most important systems that mediate communication between cells in a tissue are bioelectric signals (5), exosomes $(12,13)$ and telocytes $(14$, $15,16)$. Other possible players are allosteric interactions between proteins (17) and thermal vibrations in protein molecules (18). The role of bioelectric signals has been covered elsewhere (5). The possible roles of exosomes and telocytes have hardly been covered at all. This paper will focus on the possible role(s) of exosomes with a mention of telocytes.

\section{Theme 1. What are the details of the process that changes the epigenetic cargo of the exo- somes a cell produces when it becomes ma- lignant?}

All cells give off small lipoprotein sacks called extracellular vesicles (ECVs) that come in three varieties-exosomes (30-100 nm), microvesicles (100-1000 $\mathrm{nm}$ ) and large oncosomes (1-10 microm) (19). All contain loads of different epigenetic molecules (an 
enormous variety of proteins, several forms of RNA including miRNAs, segments of genomal DNA, lipids, and metabolites). They also possess cell-recognition proteins on their external membranes that act as keys to fit other complementary 'lock' protein molecules on the surface of their proper target molecules. Exosomes are formed inside the cell in multivesicular bodies, whereas the other two types bud off from the plasma membrane. A given cell type can construct more than one type of ECV (19). Large oncosomes are a recent discovery that derive from highly mobile and aggressive "ameboid" tumour cells (19).

A major feature of a cancer cells is that they bud off exosomes that contain a different load of epigenetic factors from that which the same cell exported before it became malignant $(20,21,22)$. The process of changing from a normal cell to a malignant one is accompanied by extensive new activity in its mechanism that selects and uploads the required molecules into the developing exosome. This process is carried out in the multivesicular bodies. This must involve inter alia the extensive switching off of some genes and the switching on of others. The details of this process are poorly known. An excellent review of the Byzantine complexities of the system has been published by Villarroya-Beltri et al. (23), and see also Minciacchi et al. (19). Microvesicles and large oncosomes have a different mechanism for cargo selection. This paper will concentrate on exosomes.

The concept of the altered morphogenetic/local field potential field of the cancer cell makes it easy to visualize how this complex co-ordinated mechanism could be modulated to change the patterns of epigenetic material packed into the developing exosome when the cell becomes malignant. These changes are very sensitive to changes in the local environment such as stress and anoxia (23). Whereas it is seems somewhat difficult to see how the single (or small number) of scattered random mutations postulated by the SMT hypothesis could do this.

But what is the function of these exosomes?

\section{Theme 2. Can exosomes produced by a ma- lignant cell induce on their own a metastatic cancer in the target tissue?}

The current orthodox account of the function of these new exosome cargoes is that, on arrival via the blood stream at their target neurons, they prepare a niche in the target cells that will allow those cells to take up the metastasized tumour cells when the latter arrive later and to provide an optimum environment for their growth thereafter (providing a new blood supply, for example). Sceneay et al. (24) put it thus,

"Primary tumor cells orchestrate pre-metastatic niche formation through secretion of a variety of cytokines and growth factors that promote mobilization and recruitment of bone marrow-derived cells to future metastatic sites. Hypoxia within the primary tumor, and secretion of specific microvesicles termed exosomes, are emerging as important processes and vehicles for tumor-derived factors to modulate pre-metastatic sites."

Hood et al. (25) state.

"Homing of melanoma exosomes to sentinel lymph nodes imposes synchronized molecular signals that effect melanoma cell recruitment, extracellular matrix deposition, and vascular proliferation in the lymph nodes. Our findings highlight the pathophysiologic role and mechanisms of an exosome-mediated process of microanatomic niche preparation that facilitates lymphatic metastasis by cancer cells."

A further series of papers have described some details of this process. In a study of exosomes from human colorectal cancer cells Li et al. (26) reported that a major finding was the selective enrichment of metastatic factors (MET, S100A8, S100A9, TNC), signal transduction molecules (EFNB2, JAG1, SRC, TNIK), lipid raft and lipid raft-associated components (CAV1, FLOT1, FLOT2, PROM1), plus a co-localization of the protein complexes EPCAM-CLDN7 and TNIK-RAP2A, in exosomes derived from metastatic SW620 cells. Peinado et al. (27) showed that exosomes from highly metastatic melanomas increased the metastatic behavior of primary tumours by permanently 'educating' bone marrow progenitors through the receptor tyrosine kinase MET. Melanoma-derived exosomes also induced vascular leakiness at pre-metastatic sites and reprogrammed bone marrow progenitors toward a pro-vasculogenic phenotype that was positive for c-Kit, the receptor tyrosine kinase Tie2 and Met.

Endometriosis has been described as a benign metastasizing disease (11). Recent evidence shows that exosome-carried miRNAs and ecto-nucleotidases play a role in its development $(28,29)$. It should also be noted that exosomes derived from tumour cells, as well as from immune cells, can also carry tumour antigens and promote immunity leading to resistance to malignant tumour development and eradication of established tumours (26).

I would like here to present an additional hypothesis. This suggests that, when some of the exosomes from malignant cancer cells arrive at their target (a tissue bearing the right surface cell recognition receptors) the cargo they release into the interior of the target cells does not simply form a niche. It seems 
possible that, in addition, the new epigenetic molecules may also corrupt the intercellular signalling between the affected cells so that they form a new local disorderly group. In which event there may be no need for that group to import a second cancer cell from the primary source, because it can grow one of its own, if the new corrupt group starts to attract, recruit and incorporate adjacent non-neoplastic cells. In other words, in the present hypothesis, all metastases must travel as entire malignant cells from the primary site to the metastatic site. Instead I suggest in some cases, in addition, its daughter exosomes may make the journey and create the new cancer cell when they reach their destination. Experiments to test this hypothesis might be to see if exosomes, isolated from a malignant cell source, when suitably applied to normal cells, could induce new cancers in them. A very recent paper reports that such an experiment has been done with results that support my hypothesis. Melo et al. (31) report that cancer exosomes process precoursor-miRNAs into mature miRNAs via a Dicer dependent mechanism. These mediate an efficient and rapid silencing of mRNAs in target cells and reprogram the target cell transcriptome with new epigenetic material. Exosomes derived from cells and sera of patients with breast cancer instigate nontumourigenic epithelial cells to form tumours in a Dicer-dependent manner (31). lows.

These hypotheses may be summarized as fol-

- Hypotheses 1. Primary tumour cells in situ orchestrate, via exosomes, the formation of pre-metastatic niches that attract metastasizing tumour cells and provide a tumour-friendly environment for their subsequent growth. There is abundant evidence for this mechanism.

- Hypothesis 2. In some cases it is possible that a travelling metastatic cancer cell could emit its own exosomes, which, on binding to a target cell, are taken up into that cell and proceed to orchestrate the local tissue into a niche. This mechanism would not need a second tumour cell to bind to form the kernel of the metastasis as the first tumour cell does that. In both these hypotheses the metastasis forms on the basis of a cancer cell moving to the new site itself. Evidence for this translocation is presented by the finding that dormant disseminated cancer cells from metastasis-free organs can re-awaken tumourigenic and metastatic capabilities if retrieved and placed in the orthotopic organ (32).

- Hypothesis 3 . In yet other cases it is possible that exosomes derived from cancer cells could be taken up into normal target cells and reprogram their genetic and epigenetic machinery to convert the normal target cell into a malignant tumour cell. A recent experiment appears to provide experimental support for this hypothesis (31). In this hypothesis the metastasis is not formed by a migrating cancer cell itself but is brought into being by exosomes from the primary tumour.

These hypotheses are not necessarily competitive and could be combined in various ways. For example, exosomes from a cancer cell in situ (acting as described in Hypothesis1) could carry in its cargo a quantity of cell specific tumour cell recognition protein molecules that it transfers to the target cell it lands on. These molecules could then transfer to the surface of this cell where they would be able lie in wait to recognize and attract a passing metastasizing cancer cell. This cell could then operate as in Hypothesis 2 and, upon binding to the niche site, release its own exosomes with cargoes that would further promote niche creation.

In these instances, these exosomes are essential for the resulting cancer to develop. Hypotheses 2 and 3 have the advantage of simplicity, as they avoid the idea that niches, organized by one set of exosomes, have to attract wandering tumour cells many of which will presumably miss their targets. Moreover this mechanism has the following problem. A niche is likely to be a dynamic construction. In the absence of occupation by a tumour cell, it would not survive for long on in the competitive environment of a cell. However, this might be circumvented by a continual rain of exosomes from the primary site, but each niche would be similarly short-lived. This process might represent an extravagant use of the cell's resources. The problem is avoided if the exosomes from one tumour cell engineer both the malignant transformation of the target cell and construct the niche for it, as my hypothesis suggests.

In support of the suggestion that exosome epigenetic loads can engineer extensive changes in target cells, it is noteworthy that a similar situation occurs in the nervous system. Here the modality of a neuron, and details of its operational connectivity network, are determined, not by where in the brain it is located, but whence its afferent nerve supply originates (13). In the sensory system, for example, if the normal afferent supply to the visual cortex is cut and surgically replaced by input from the afferent somatosensory system, the visual neurons involved are subjected to extensive microanatomical and neurophysiological reorganization, and cease to function as visual neurons and function as somatosensory neurons instead. This is shown by experiments on blind subjects expert in Braille (33). In these, if the correct part of the visual cortex is stimulated by transcranial magnetic pulses the subject does not see the flashes of light that a normal person would experience, but feels a touch on 
the finger instead. This shows that in the blind subject the now inactive visual neurons are taken over and used by neurons of the adjacent somatosensory cortex.

\section{Theme 3. What is the functional significance of the fact that exosomes from cancer cells carry in their loads segments of genomal DNA bearing cancer-related mutations across the entire spectrum?}

The SMT and TOFT hypotheses may be partly united by the following observation. In cases of human pancreatic cancer, Kahlert et al. (34) report that exosomes released from cancer cells contain $>10-\mathrm{kb}$ fragments of double-stranded genomic DNA. In these fragments they detected mutations in KRAS and p53. KRAS and p53 are the two most frequent mutated genes in pancreatic ductal cancer. The mutations found in this study in the exosomal DNA were a KRAS mutation on codon 12 (GGT->TGT) and a second KRAs mutation on codon 22 (CAG $->C T G)$. A third p53 mutation was on codon 273 (CGT ->CAT). Thus exosomes from these cancer cells were carrying mutations identical to their parent cancer cells. In addition, using whole genome sequencing, they demonstrated that serum exosomes from these patients contained genomic DNA spanning all chromosomes. The authors then proceed to discuss the clinical applications of their findings as diagnostic tools. They do not discuss them in the context of relating to theories of the mechanism of cancer neogenesis. So what could be the functional effect in the target cell of the transport into it of the DNA cancer-related genetic mutations themselves?

\section{Theme 4. What is the evolutionary advantage for the organism of the production by its can- cer cells of exosomes that carry epigenetic instructions for the building of elaborate mo- lecular mechanisms that promote the growth of metastatic cancers?}

The classical hypothesis states that the only function of exosomal transported epigenetic molecules is specifically to prepare the niches for reception of the metastatic cancer cells. This seems to have no evolutionary relevance. Normal cells do not enter the blood stream and travel to distant organs there to enter the tissue. But they do form exosomes, which enter the blood and locate in distant organs. But, in these new locations, they do not go in for niche construction. There is no normal function in this account for the cancer process to subvert. So why do exosomes from cancer cells build niches? Our supplementary hypothesis suggests that these exosomes have the additional function of forming new metastases them- selves. Could this be regarded as an unavoidable side effect of the normal role for these exosomes, which is to carry functionally active information between normal cells?

\section{Telocytes and cancer}

Telocytes (TCs) form a remarkable new cell species found in many types of tissue $(14,15,16)$. They are characterized by having very small cell bodies and extremely long and thin tubular processes called telopodes (up to 100 micrometers long, yet only 20-200 nanometers wide). Telopodes consist of long thin tubes (called podomers) interspersed with short dilations (called podoms) that have the appearance of axonal boutons en passage. Podoms contain abundant mitochondria, calveoli and endoplasmic reticulum. TCs form a dense convoluted network linking TCs with each other, and with many other cell types including other telocytes and stem cells with which the exchange exosomes. They are thought to act as integrators of many intercellular functions. A literature search discovered only two papers on this subject. In tissue culture experiments on the self-assembly of reconstituted breast cancer tissue Mou et al. (35) showed that telocytes promoted the formation of typical breast structure self-assembly, promoted the proliferation of breast cancer cells, and inhibited their apoptosis. Telocytes in skin in cases of basal cell and squamous cell carcinoma have a greatly reduced number of heterocellular contacts (36). Thus telocytes may also be involved in cancer development but clearly many more investigations need to be carried out.

\section{Conclusions}

- the data presented relating to theme 1 may be more easily explained by the TOFT than the SMT theory.

-following the lead given by Melo et al. (31), further experiments to test the hypothesis developed by theme 2 -that exosomes from cancer cells can, by themselves, induce new cancers in normal cells - need to carried out.

- the data presented by Kahlert et al. (34) under theme 3 needs to be evaluated with reference to its significance for theories of cancer neogenesis.

- the issue raised by theme 4 needs further elucidation.

It may be possible that there are two types of metastasis-related niche. One is produced by exosomes produced by the primary tumour in situ. The second is produced by exosomes from traveling cancer cells that have already invaded the target tissue. Thus this hypothesis may throw new light on the effect these exosomes have on the normal cells they 
invade. Previously the idea was that the only function of the epigenetic loads of the exosomes, emitted by cancer cells, was to prepare a suitable environment in the target cell for something else-i.e. another cancer cell-or possibly a fragment of double-stranded genomenal DNA with mutated bases in it-to develop and grow. Whereas, what really maybe happening might be that the epigenetic reprogramming of the target cell's biochemistry by exosomes from either of these two sources constitutes the metasasis.

The estimation of the validity of this hypothesis needs to be based on further experiments.

\section{Acknowledgement}

I am grateful to Michael Levin for introducing me to this topic and for his most helpful comments.

\section{Conflicting interests}

I have no conflicting interests.

\section{References}

1. Waddington $\mathrm{CH}$. Cancer and the theory of organizers. Nature $1935 ; 135$ : 606-608. doi:10.1038/135606a0.

2. Burr HS. Biological organization and the cancer problem. Yale J Biol Med 1940; 12: $277-82$.

3. Rubin H. Cancer as a dynamic developmental disorder. Perspect Cancer Res 1985; 45: 2935-42.

4. Sotto AM, Sonnenschein C. The tissue organization field theory of cancer: A testable replacement for the somatic mutation theory. Bioessays 2011; 33: 332-3. doi: 10.1002/bies.201100025.

5. Chernet B, Levin M. Endogenous Voltage Potentials and the Microenvironment: Bioelectric Signals that Reveal, Induce and Normalize Cancer. J Clin Exp Oncol 2013; S1. doi:10.4172/2324-9110.S1-002.

6. Baker SG. A cancer theory kerfuffle can lead to new lines of research. J Natl Cancer Inst. 2015; doi: 10.1093/jnci/dju405.

7. Blackiston D1, Adams DS, Lemire JM, Lobikin M, Levin M. Transmembrane potential of $\mathrm{GlyCl}$-expressing instructor cells induces a neoplastic-like conversion of melanocytes via a serotonergic pathway. Dis Model Mech 2011; 4 : 67-85. doi: 10.1242/dmm.005561.

8. Taylor IW, Linding R, Warde-Farley David, Liu Y, Pesquita C, Faria D, Bull S, Pawson T, Morris Q, Wrana JL. Dynamic modularity in protein interaction networks predicts breast cancer outcome. Nature Biotechnology 2009; 27: 199-204 . doi:10.1038/nbt.1522.

9. Dokukin ME, Guz NV, Gaikwad RM, Woodworth CD, Sokolov I Cell Surface as a Fractal: Normal and Cancerous Cervical Cells Demonstrate Different Fractal Behavior of Surface Adhesion Maps at the Nanoscale. Phys Rev Lett 2011; 107: 028101.

10. Dokukin ME, Guz NV, Woodworth CD, Sokolov I. Emergence of fractal geometry on the surface of human cervical epithelial cells during progression towards cancer. New J Phys 2015; 17: 033019. doi:10.1088/1367-2630/17/3/033019.

11. Tarin D. Cell and tissue interaction in carcinogenesis and metastasis and their clinical significance. Semin Cancer Biol 2011; 21: 72-82.

12. Edelstein $\mathrm{L}$ and Smythies J. "The role of the epigenetic codes in neurocomputation: dynamic hardware in the brain". In Edelstein L, Smythies J, Noble D. Co-editors. Theme Issue "Epigenetic information-processing mechanisms in the brain." 2014c Phil. Trans. R. Soc. B. 2014; 369: 1652.

13. Smythies J, Edelstein L. Transsynaptic signaling codes in the brain and their significance: possible involvement of synchronized spike codes, large molecular transsynaptic signaling agents and exosomes. Front Integ. Neurosci. 2012; 6:126. doi: 10.3389/fnint.2012.00126.

14. Smythies J, Edelstein L. Telocytes, exosomes, gap junctions and the cytoskeleton: the makings of a primitive nervous system? Front. Cell Neurosci. 2013; doi: 10.3389 /fncel.2013.00278

15. Edelstein L and Smythies J. Epigenetic aspects of telocytes/cordocytes: jacks of all trades, masters of most. Front Cell Neurosci. 2014; 8:32. doi: 10.3389/fncel.2014.00032.

16. Edelstein L, Smythies J. The role of telocytes in morphogenetic bioelectrical signaling: once more unto the breach. Front Mol Neurosci 2014; 7: 41. doi: 10.3389/fnmol.2014.00041.

17. Fuxe K, Borroto-Escuela DO, Ciruela F, Guidolin D and Agnati LF. Receptor-receptor interactions in heteroreceptor complexes: a new principle in bi- ology. Focus on their role in learning and memory. Neurosci Discov 2014; 2: 6. Doi: $10.7243 / 2052-6946-2-6$.

18. Smythies J. On the role of protein vibrations in information processing in the brain: three Russian dolls. In preparation. 2015

19. Minciacchi VR, Freeman MR, Di Vizio D. Extracellular Vesicles in Cancer: Exosomes, Microvesicles and the Emerging Role of Large Oncosomes. Semin Cell Dev Biol 2015; doi: 10.1016/j.semcdb.2015.02.010.

20. Zhang HG, Grizzle WE. Exosomes: a novel pathway of local and distant intercellular communication that facilitates the growth and metastasis of neoplastic lesions. Am J Pathol 2014;184: 28-41. doi: 10.1016/j.ajpath.2013.09.027.

21. Kosaka N, Yoshioka Y, Tominaga N, Hagiwara K, Katsuda T, Ochiya T. Dark side of the exosome: the role of the exosome in cancer metastasis and targeting the exosome as a strategy for cancer therapy. Future Oncol 2014; 10: 671-81. doi: 10.2217 / fon.13.222.

22. D'Asti E, Garnier D, Lee TH, Montermini L, Meehan B, Rak J. Oncogenic extracellular vesicles in brain tumor progression. Front Physiol. 2012; 3: 294. doi: 10.3389/fphys.2012.00294.

23. Villarroya-Beltri C, Baixauli F, Gutiérrez-Vázquez C, Sánchez-Madrid F, Mittelbrunn M. Sorting it out: regulation of exosome loading. Semin Cancer Biol 2014; 28: 3-13. doi: 10.1016/j.semcancer.2014.04.009.

24. Sceneay J, Smyth MJ, Möller A. (2013) The pre-metastatic niche: finding common ground. Cancer Metastasis Rev 2013; 32: 449-64. doi: 10.1007/s10555-013-9420-1.

25. Hood JL, San RS, Wickline SA. Exosomes released by melanoma cells prepare sentinel lymph nodes for tumor metastasis. Cancer Res 2011; 71:3792-801. doi: 10.1158/0008-5472.CAN-10-4455.

26. Ji H, Greening DW, Barnes TW, Lim JW, Tauro BJ, Rai A, Xu R, Adda C, Mathivanan S, Zhao W, Xue Y, Xu T, Zhu HJ, Simpson RJ. Proteome profiling of exosomes derived from human primary and metastatic colorectal cancer cells reveal differential expression of key metastatic factors and signal transduction components. Proteomic 2013; 13:1672-86. doi: 10.1002/pmic.201200562.

27. Peinado H, Alečković M, Lavotshkin S, Matei I, Costa-Silva B, Moreno-Bueno G, Hergueta-Redondo M, Williams C, García-Santos G, Ghajar C, Nitadori-Hoshino A, Hoffman C, Badal K, Garcia BA, Callahan MK, Yuan J, Martins VR, Skog J, Kaplan RN, Brady MS, Wolchok JD, Chapman PB, Kang Y, Bromberg J, Lyden D. Melanoma exosomes educate bone marrow progenitor cells toward a pro-metastatic phenotype through MET. Nat Med 2012; 18: 883-91. doi: $10.1038 / \mathrm{nm} .2753$.

28. Gilabert-Estelles J, Braza-Boils A, Ramon LA, Zorio E, Medina P, Espana F, Estelles A. (2012) Role of microRNAs in gynecological pathology. Curr Med Chem 2012; 15: 2406-13. doi.org/10.2174/092986712800269362.

29. Texidó L, Romero C, Vidal A, García-Valero J, Fernández Montoli ME, Baixeras N, Condom E, Ponce J, García-Tejedor A, Martín-Satué M. Ecto-nucleotidases activities in the contents of ovarian endometriomas: potential biomarkers of endometriosis. Mediators Inflamm 2014; 120673. doi: $10.1155 / 2014 / 120673$.

30. Greening DW, Gopal SK, Xu R, Simpson RJ, Chen W. Exosomes and their roles in immune regulation and cancer. Semin Cell Dev Biol; 2015; doi: 10.1016/j.semcdb.2015.02.009.

31. Melo SA, Sugimoto H, O'Connell JT, Kato N, Villanueva A, Vidal A, Qiu L, Vitkin E, Perelman LT, Melo CA, Lucci A, Ivan C, Calin GA, Kalluri R. Cancer exosomes perform cell-independent microRNA biogenesis and promote tumorigenesis. Cancer Cell 2014; 26: 707-21. doi: 10.1016/j.ccell.2014.09.005.

32. Suzuki M, Mose ES, Montel V, Tarin D. Dormant cancer cells retrieved from metastasis-free organs regain tumorigenic and metastatic potency. Am J Pathol 2006; 169: 673-81.

33. Ptito M, Fumal A, de Noordhout AM, Schoenen J, Gjedde A, Kupers R. TMS of the occipital cortex induces tactile sensations in the fingers of blind Braille readers. Exp Brain Res 2008; 184: 193-200. doi:10.1007/s00221-007-1091-0.

34. Kahlert C, Melo SA, Protopopov A, Tang J, Seth S, Koch M, Zhang J, Weitz J, Chin L, Futreal A, Kalluri R. . Identification of double-stranded genomic DNA spanning all chromosomes with mutated KRAS and p53 DNA in the serum exosomes of patients with pancreatic cancer. J Biol Chem. 2014; 289: 3869-75. doi: 10.1074/jbc.C113.532267.

35. Mou Y, Wang Y, Li J, Lü S, Duan C, Du Z, Yang G, Chen W, Zhao S, Zhou J, Wang C. Immunohistochemical characterization and functional identification of mammary gland telocytes in the self-assembly of reconstituted breast cancer tissue in vitro. J Cell Mol Med 2012; 17: 65-75. doi: 10.1111/j.1582-4934.2012.01646.x.

36. Mirancea N, Moroşanu AM, Mirancea GV, Juravle FD, Mănoiu VS. Infrastructure of the telocytes from tumor stroma in the skin basal and squamous cell carcinomas. Rom J Morphol Embryol 2013; 54: 1025-37. 